\title{
Behavioral strategy shifts with training: Rats on an elevated plus maze
}

\author{
JENNIFER TROPP and ETAN J. MARKUS \\ University of Connecticut, Storrs, Connecticut
}

\begin{abstract}
Rats use a multitude of cues within the environment to guide their behavior. The purpose of this study was to investigate the degree to which reliance on local cues, visual extramaze cues, and other sources of information changes with training. Female Fischer 344 rats were trained consecutively for 24 days on a four-arm radial maze reference memory task. The correct arm could be located by its relation to the "outside world," a large white illuminated poster, or to a local insert on the goal arm. Within 2 weeks, the animals were consistently choosing the goal arm regardless of how they were placed on the maze. Four and 10 days after reaching asymptotic performance, a probe trial was given to determine what strategy the animals were using to solve the task. During the probe trial, both the visual extramaze cue and the local cues were rotated $90^{\circ}$ clockwise and counterclockwise, respectively, and the animals' choice of arm was recorded. This probe revealed which cues had the greatest influence on each animal's behavior. Initially, the animals tended to use all three types of cues equally in solving the task. With additional training, there was a shift toward predominantly using visual extramaze information and ignoring information outside of the testing environment. Thus, the use of environmental information is dynamic, even within a specific task, and it changes with the extent of the training.
\end{abstract}

Animals use various cues in order to navigate through their environment. For example, homing pigeons use the position of the sun while foraging (Gallistel, 1990), bats use echo-location (Gallistel, 1990), and ants shift between the use of olfactory, celestial, and visual landmark information to navigate their way (Hölldubler \& Wilson, 1990). In their natural environment, rats show flexibility in their mode of navigation and can navigate successfully even in the absence of any observable cues (Calhoun, 1962). Rats can use landmarks, local sensory information, distal information, and "dead reckoning" to guide their behavior (e.g., McNaughton, Chen, \& Markus, 1991). One can infer the preference of an animal to use one form of navigation over another by examining the rate of learning on tasks that differ in their navigational requirements. Thus, for example, rats learn appetitive tasks based on spatial location (e.g., using visual or extraroom cues) faster than those based on cues local to the maze (e.g. Jarrard, 1993; Oler \& Markus, 1998; Ward, Stoelzel, \& Markus, 1999). Another approach is to limit the use of specific types of information and observe whether the animals can still learn the task. For example, preventing rats from utilizing extraroom and/or vestibular information impairs learning of a spatial radial arm maze task (Dudchenko, Goodridge, Seiterle, \& Taube, 1997; Martin, Harley, Smith, Hoyles, \& Hynes, 1997).

The present research was supported in part by UCONN Research Foundation, Grant 441713, and NSF Grant IBN 9809958. We thank Carl R. Stoelzel for his assistance with the animal training. Address correspondence to E. J. Markus, Behavioral Neuroscience Division, Department of Psychology, University of Connecticut, 406 Babbidge Rd. Box U-20, Storrs, CT 06269 (e-mail: markus@psych.psy.uconn.edu).
Stronger evidence for an animal's biases in using environmental information can be obtained from experiments in which different types of cues provide conflicting information to the animal. The animal is trained to go to a certain location in a stable environment. Once the task has been learned, a probe trial is conducted in which different aspects of the task are manipulated. The animal's response provides an index of which cues are most influential in guiding its behavior. For example, probe trials have revealed that as rats age, they tend to rely less on distant visual landmarks and more on local sensory information (e.g., Oler \& Markus, 1998) or the use of a motor response strategy (Barnes, Nadel, \& Honig, 1980). Interestingly, the amount of training has also been found to affect which cues the animal relies on. A number of studies have shown that with extended training, animals shift from displaying location-based learning to motorresponse-based learning (Hicks, 1964; Packard \& McGaugh, 1996; Ritchie, Aeschliman, \& Pierce, 1950).

In the studies mentioned above, the subjects were male rats, and only spatial and motor response strategies were compared. In the present study, we examined whether (1) female rats relied more on a visual extramaze cue, local sensory cues, or extraroom sources of information to find a designated food reward, and (2) whether animals change their reliance on these different types of environmental cues with extended training.

\section{METHOD}

\author{
Subjects \\ Twenty-two 8-month-old female Fischer 344 retired breeders \\ (Harlan Sprague-Dawley, IN) were singly housed in transparent
}


plastic tubs in a room with a 12:12-h light:dark cycle (lights off at $1800 \mathrm{~h}$ ). All animals underwent extensive handling during the month preceding training.

\section{Apparatus}

The maze consisted of four black Plexiglas arms $(10.5 \times 51 \mathrm{~cm})$ forming a symmetrical " $X$ " (see Figure la). The testing room $(2.1 \times 2.1 \mathrm{~m})$ was completely black except for an illuminated white cue card $(123 \times 243 \mathrm{~cm})$ located on the south $(\mathrm{S})$ wall, approximately $53 \mathrm{~cm}$ from the edge of the two closest maze arms. The initial segments of the arms differed in their tactile, visual, and, possibly, olfactory characteristics. Three of the arms had an insert: a black piece of rubber $(20 \times 10 \mathrm{~cm})$, black wire mesh $(25 \times 10 \mathrm{~cm})$, or white contact paper $(25 \times 10 \mathrm{~cm})$. The fourth arm was uncovered (i.e., black Plexiglas surface). Extraroom cues included the single entryway, and auditory stimuli (e.g., computer fans) from outside the maze room. Throughout the experiment only the southwest (SW) arm, with a rubber insert, was baited with chocolate sprinkles. The maze was cleaned with alcohol before each rat was run.

\section{Procedure}

After food deprivation to $85 \%$ of their ad-lib weights, the animals were trained to go to the SW arm. Every morning the animals were transported from the animal room to the maze room in their home cages, always along the same pathway and through the same entryway. The rat was placed in the center of the maze. Once it traversed down the baited arm, it was picked up and placed back on the center of the maze. In order to prevent the development of a motor response strategy, each time the animal was put on the maze it was placed facing a different arm. ' After the first visit to the baited arm, the food cup was rebaited and this procedure was repeated five times or until 10 min elapsed.

From the fifth day forward, the morning session was terminated early if the animal visited an incorrect arm. Thus, in a given morning session, a rat was scored from 0 to 5 , depending on its behavior $(0=$ error on first arm visited $; 5=$ consistently visited the correct arm on all five trials). To constitute a visit, the rat's front forelimbs had to pass $24 \mathrm{~cm}$ (i.e., about halfway) down the arm. All animals were also retested in an afternoon session in which the animal was taken off the maze after visiting a single arm regardless of its correctness (i.e., the rat was rewarded only if it visited the SW arm). The afternoon sessions were conducted to familiarize the animals with a single-trial procedure.

On Day 18, a probe session was conducted in which the afternoon session was modified. The local inserts were rotated $90^{\circ}$ counterclockwise, the illuminated white cue card was rotated $90^{\circ}$ clockwise, and all four arms were baited (see Tanila, Shapiro, Gallagher, \& Eichenbaum, 1997). The animals were placed on the maze facing the NE arm (see Figure lb). This allowed for a dissociation among animals predominantly relying on the local inserts ( $\mathrm{arm} \mathrm{SE}$ ), the visual extramaze cue ( $\operatorname{arm} \mathrm{NW}$ ), and extraroom information ( $a r m \mathrm{SW}$ ), in guiding their behavior. The choice of the $\mathrm{NE}$ arm could indicate that the animal was using some other means to guide its behavior (e.g., it simply continued down the arm it was facing). It should be noted that the animals were previously familiarized with the single-trial afternoon procedure and that their choice was reinforced regardless of the arm selected. Therefore, the probe session was designed to provide minimal disruption to ongoing performance. Regular morning and afternoon sessions continued for 6 more days at which time the probe session was repeated.

\section{RESULTS}

A repeated measures analysis of variance showed significant learning over training days $[F(23,483)=51.97$, $p<.01$ ] during the morning sessions (Figure 2a). By approximately Day 14 , the rats reached asymptotic levels of behavior (consistently visiting the correct arm). Thus, the animals were reliably choosing the baited arm regardless of how they were placed on the maze. Importantly, this level of performance was stable and unaffected by the afternoon probe, with the animals displaying a high level of accuracy both before and after the first probe (Figure 2a). The probes were conducted on Day 18 and

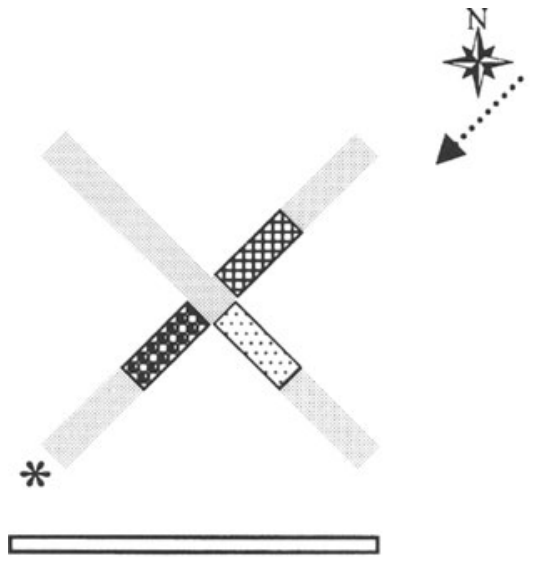

(a)
Standard

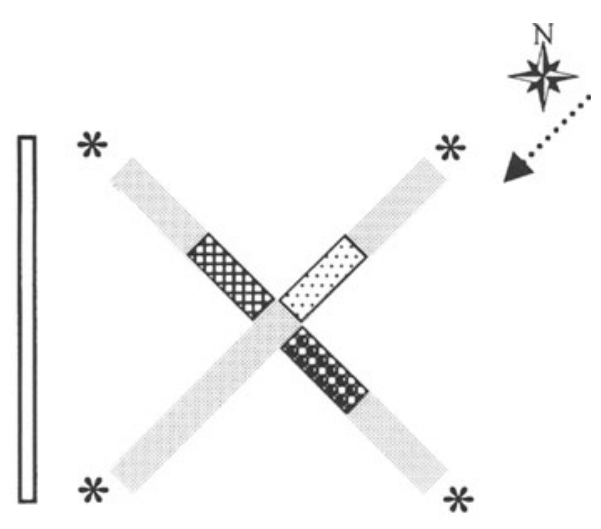

(b)

\section{Probe}

Figure 1. Standard and probe configuration of maze and testing room: (a) The room was completely black except for an illuminated white cue card located alongside the south wall. Three of the arms had a distinct type of insert. In the standard sessions, only the southwest arm with the rubber insert was baited. (b) During the probe sessions, the inserts were rotated $90^{\circ}$ counterclockwise, the visual cue was rotated $90^{\circ}$ clockwise, and all of the arms were baited. In both the standard and probe conditions, the animals were always brought in from the same entryway (dashed arrow). *A baited arm. 


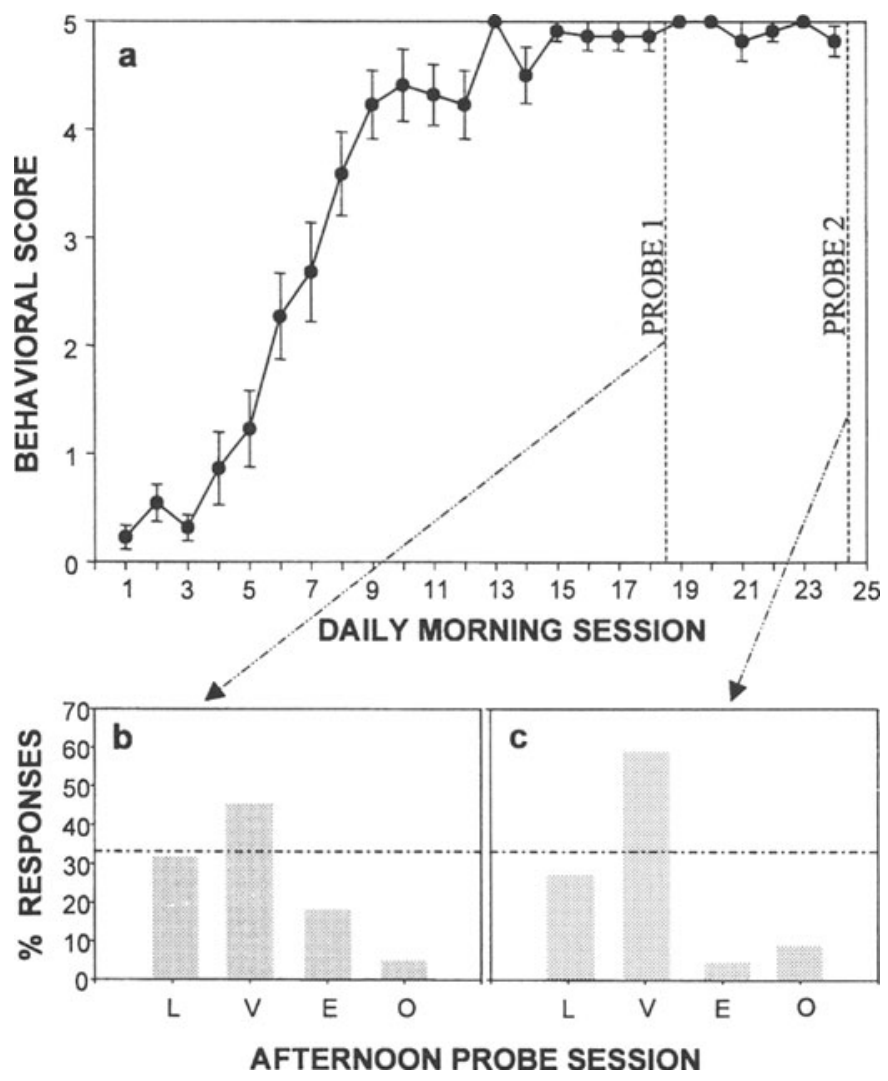

Figure 2. (a) Behavioral score for the daily morning sessions: The number of times each rat chose the baited arm before making an error (maximum of five trials per session). (b) and (c) Probe sessions: Every afternoon the animals were given a single trial and were taken off the maze. On the afternoon of Day 18 and Day 24, all arms were baited and a special probe session was conducted. The local inserts were rotated $90^{\circ}$ counterclockwise and the visual cue rotated $90^{\circ}$ clockwise. (b) First probe session (Day 18): Percent of animals that relied on the local cues $(L)$, visual cue $(V)$, extraroom cues $(E)$, or other sources $(O)$. There was no significant preference among the three cue strategies. (c) Second probe session (Day 24): Percent of animals that relied on the local cues (L), visual cue (V), extraroom cues (E), or other sources $(O)$. There were significant differences among the three cue strategies, with an overall bias for the visual cue. The dashed line (at $33 \%$ ) is the expected proportion of responses if animals rely on all three types of cues to an equal degree.

Day 24, approximately 4 and 10 days (respectively) after the animals exhibited near perfect performance.

As in the morning sessions, within 2 weeks the animals were proficient during the afternoon sessions. This was apparent through the observation that no errors were committed on the afternoon session by any of the animals on the 4 days proceeding each of the probes. The latency to choosing an arm was likewise very short, averaging $2.6 \mathrm{sec}(S E M=0.5 \mathrm{sec})$ on the last 4 days preceding the first probe, and $2.1 \mathrm{sec}(S E M=0.6 \mathrm{sec})$ on the last 4 days preceding the second probe. In contrast to the rapid choices on the regular afternoon sessions, during the two probe sessions the animals took much longer to choose an arm,
$11.4 \mathrm{sec}(S E M=2.5 \mathrm{sec})$ for the first probe and $10.5 \mathrm{sec}$ $(S E M=2.4 \mathrm{sec})$ on the second probe trial.

A $\chi^{2}$ analysis was performed for both afternoon probe sessions in order to determine whether there was a significant tendency to use a particular type of cue in guiding behavior. On the first probe (Figure $2 \mathrm{~b}$ ) rats showed no preference among the three cue strategies $\left[\chi^{2}(2, N=\right.$ $21)=2.57, p>.25]$. However, on the second probe (Figure $2 \mathrm{c})$, there were significant differences $\left[\chi^{2}(2, N=\right.$ $20)=10.90, p<.01]$, in which most animals relied on the visual extramaze cue to guide their behavior.

In many cases animals start down one particular arm and then turn around and proceed down another (see 
Brown, 1993). These partial or initial responses were operationally defined as proceeding half a body length down an arm. ${ }^{2}$ Although an animal may make many of these partial responses before making a final decision (and eating the food), only the first of these responses was recorded. A $\chi^{2}$ analysis was performed for both afternoon probe sessions in order to determine whether there was a tendency to use a particular type of cue in the rats' initial response. On the first probe there was a significant difference in the initial responses $\left[\chi^{2}(2, N=21)=8.0\right.$, $p<.02]$, with animals responding mostly to the local cues (Figure 3a). There was also a significant difference in the initial responses during the second probe $\left[\chi^{2}(2, N=21)=\right.$ $6.0, p<.05$ ], with animals relying mostly on the local or visual extramaze cues rather than on the extraroom cues (Figure 3c). As can be seen in Figures $3 \mathrm{~b}$ and $3 \mathrm{~d}$, the initial response did not always predict the animal's final choice. All animals that started down the visually cued arm continued down to the end of the arm. Conversely, a large proportion of the animals that initially started down an arm on the basis of local or extraroom cues made a final choice on the basis of the visual extramaze cue.

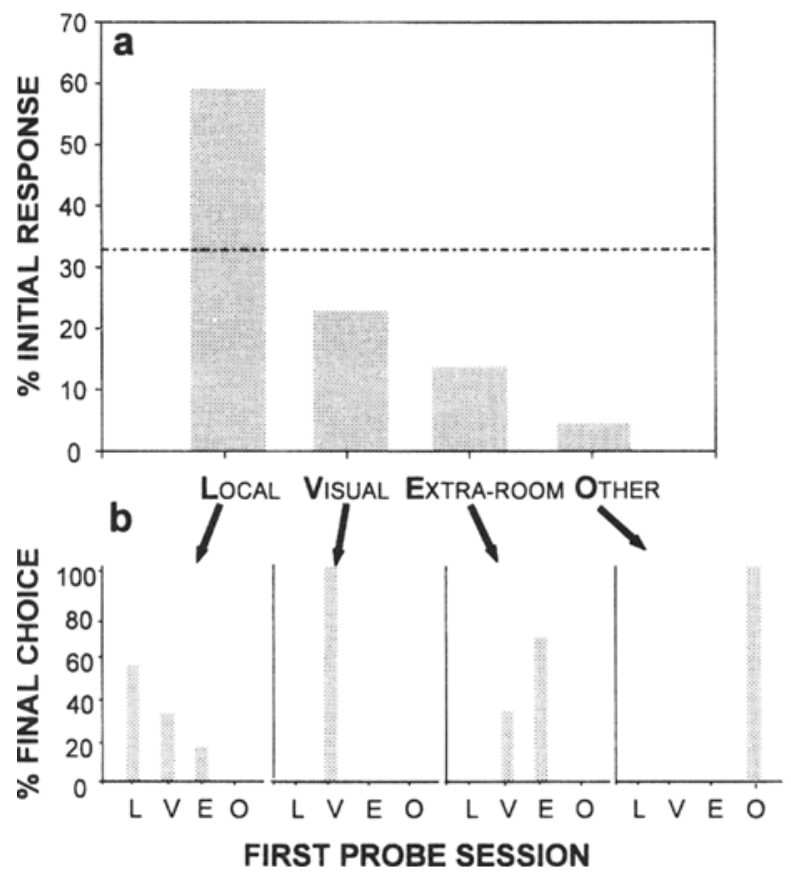

\section{DISCUSSION}

The rats were trained on a reference memory task that could be solved in three different ways. The correct arm could be located by its relation to the "outside world," a large illuminated poster, or to a local insert on the goal arm. The animals learned the task within 2 weeks and were consistently choosing the goal arm regardless of how they were placed on the maze. This level of performance was stable and unaffected by the afternoon probes. The probes were conducted approximately 4 and 10 days after the animals exhibited near perfect performance. During the probes, the three types of cues were dissociated, each aligned with a different "correct" goal arm. Notably, during the probes the rats did not always proceed down the arm initially selected, and they took much longer to make a decision, supporting the interpretation that there was a conflict between these three different strategies in individual animals.

During the first probe there was no significant preference for any of the three arms - that is, no reliance on any specific type of cue. In contrast, during the second

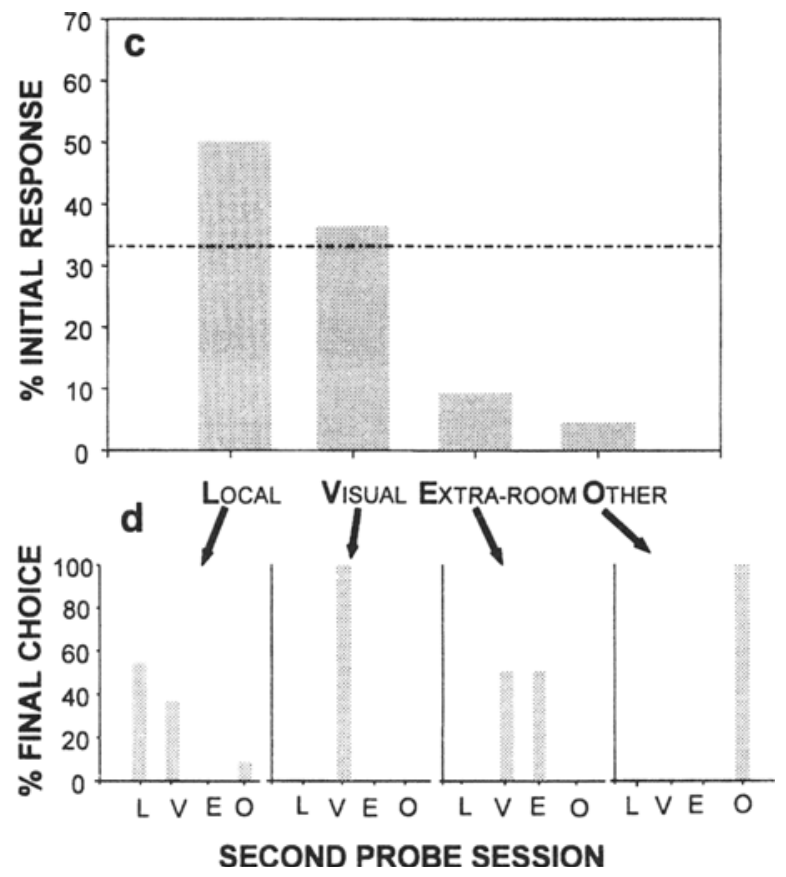

Figure 3. (a) Initial responses for the first probe session (Day 18): Percent of animals giving initial responses in relation to the different cues. The first choice for each animal was recorded. There were significant differences among the three possible cued responses, with an overall bias for the local cues. (b) Final choices for the first probe session: The subgraphs represent the breakdown of the choices in relation to the initial response. A large proportion of the animals with an initial bias toward local or extramaze cues made a final choice on the basis of the visual cue. (c) Initial responses for second probe session (Day 24): Percent of initial responses in relation to the available cues. There were significant differences among the three possible cued responses, with an overall bias to ignore the extramaze information. (d) Final choices for the second probe session: The subgraphs represent the breakdown of the choices in relation to the initial response. A large proportion of the animals with an initial bias toward local or extramaze cues made a final choice based on the visual cue. $L$, local cues; $V$, visual cues; $E$, extraroom cues; $O$, other sources. 
probe a majority of rats relied on the visual extramaze cue in choosing the goal arm, with little (if any) impact of the extraroom information.

These data suggest that during early exposure to a novel environment, rats attend to, and utilize, a wide array of information. However, with additional experience there is a tendency to focus primarily on distal visual landmarks. Rats can be trained to use a variety of cues, such as tones, odors, motor responses, and so forth, to solve a given task. The present results indicate that even when rats are not trained to solve a problem in a specific manner, they will develop a preferred way of solving the task.

Clearly the relative salience of the different types of cues plays a critical role in the degree to which an animal will rely on them (Rescorla \& Wagner, 1972). Presumably, another configuration of more or less salient cues would have a different effect on the animals. The present findings showed that with extended training the influence of the visual extramaze cue increased, and that the role of the extraroom information diminished. These results may indicate that as a rat becomes more familiar with the environment, stable distal landmarks tend to exert a stronger influence on its behavior. An alternative interpretation of these data is that overshadowing occurs (Pavlov, 1927) - that preexisting weak biases in cue utilization get stronger. Thus in the present experiment, early during training (Probe 1) there was a weak tendency to preferentially rely on the visual extramaze cue. With additional training, this initial tendency got stronger (Probe 2). However, this alternative explanation seems unlikely, given the fact that the individual rats that chose arms aligned with the extraroom or local cues early during training (Probe 1) changed their preference on the second probe. Furthermore, during the first probe about $70 \%$ of the animals started down the arm cued by the local insert. Despite the strong influence of the local insert, the visual extramaze cue became the most influential with extended training. Thus it seems that it was the type of information provided (stable distal landmark) rather than only the salience of the cue that was the important factor.

Rats can solve land mazes either by learning motor response patterns or by using local sensory and extramaze information. The present experiment constrained the use of motor responses and forced the animals to use environmental information. Furthermore, the paradigm used allowed for a dissociation between spatial information within and external to the testing environment. The results show that early during training the animals used all three types of information. With extended training, the animals tended to stop using extraroom information and focused more on the distal information within the testing environment. Although the present experiment is the first to show changes in spatial-environmental cue utilization with training, others have shown that with ex- tended training rats exhibit changes in their tendency to use a motor response over a spatial one (Hicks, 1964; Packard \& McGaugh, 199633 ;itchie et al., 1950). These studies showed a shift from spatial to motor response strategy in male rats, whereas the present study shows changes in cue utilization with training in female rats. It may be of interest in the future to examine for sex differences in the dynamics of cue use during navigational behavior.

Overall, the results have important implications for research relating brain and behavior. Research into the behavioral effects of pharmacological manipulations, aging, or the neuronal mechanisms underlying navigation must account for the extent of the animals' training. For example, Oler and Markus (1998) showed that old male rats tend to rely on information local to the maze, whereas young rats attend more to visual extramaze information. These data were interpreted as a difference in spatial ability; alternatively they may reflect age differences in cue utilization over time. Early during training, all animals may be using the local cues; however, the shift toward using the visual extramaze cues may take longer in the old animals. Possibly, if they had examined the animals after a longer training period, they would not have found age differences.

Rats use a variety of means to navigate through the environment. Many variables affect the specific type of information that the animal relies on. These include the relative salience, availability of stimuli, task demands, and species-specific tendencies to rely on certain types of cues. The present findings indicate not only that are there differences in which type of information is utilized in a particular situation, but that the use of environmental information is dynamic and changes with exposure to the environment.

\section{REFERENCES}

Barnes, C. A., NAdel, L., \& Honig, W. K. (1980). Spatial memory deficit in senescent rats. Canadian Journal of Psychology, 34, 29-39.

Brown, M. F. (1993). Sequential and simultaneous choice processes in the radial-arm maze. In T. R. Zentall (Ed.), Animal Cognition: A tribute to Donald A. Riley (pp. 153-173). Hillsdale, NJ: Erlbaum.

Calmoun, J. B. (1962). The ecology and sociology of the Norway rat (pp. 71-82). Bethesda, MD: U.S. Department of Health, Education, \& Welfare.

Dudchenko, P. A., Goodridge, J. P., Seiterle, D. A., \& Taube, J. S. (1997). Effects of repeated disorientation on the acquisition of spatial tasks in rats: Dissociation between the appetitive radial arm maze and aversive water maze. Journal of Experimental Psychology: Animal Behavior Processes, 23, 194-210.

Gallistel, C. R. (1990). The organization of learning. Cambridge, MA: MIT Press.

Hicks, L. H. (1964). Effects of overtraining on acquisition and reversal of place and response learning. Psychological Reports, 15, 459-462.

Hölldubler, B., \& Wilson, E. O. (1990). The ants. Cambridge, MA: Harvard University Press.

JARRARD, L. E. (1993). On the role of the hippocampus in learning and memory in the rat. Behavioral \& Neural Biology, 60, 9-26.

Martin, G. M., Harley, C. W., Smith, A. R., Hoyles, E. S., \& Hynes, 
C. A. (1997). Spatial disorientation blocks reliable goal location on a plus maze but does not prevent geal location in the Morris maze. Journal of Experimental Psychology: Animal Behavior Processes, 23, 183-193.

McNaughton, B. L., Chen, L. L., \& Markus, E. J. (1991). "Dead reckoning," landmark learning, and sense of direction: A neurophysiological and computational hypothesis. Journal of Cognitive Neuroscience, 3, 191-201.

Oler, J. A., \& Markus, E. J. (1998). Age-related deficits on the radial maze and in fear conditioning: Hippocampal processing and consolidation. Hippocampus, 8, 402-415.

PaCKard, M. G., \& MCGaugh, J. L. (1996). Inactivation of hippocampus or caudate nucleus with lidocaine differentially affects expression of place and response learning. Neurobiology of Learning \& $\mathrm{Mem}$ ory, 65, 65-72.

Pavlov, I. P. (1927). Conditioned reflexes (G. V. Anrep, Trans.). London: Oxford University Press.

Rescorla , R. A., \& WAGNer, A. R. (1972). A theory of Pavlovian conditioning: Variations in the effectiveness of reinforcement and nonreinforcement. In A. H. Black \& W. F. Prokasy (Eds.) Classical conditioning II: Current research and theory (pp. 64-99). New York: Appleton-Century-Crofts.

Ritchie, B. F., Aeschliman, B., \& Pierce, P. (1950). Studies in spatial learning: VIII. Place performance and the acquisition of place dispositions. Journal of Comparative Physiological Psychology, 43, 73-85.
Tanila, H., Shapiro, M., Gallagher, M., \& Eichenbaum, H. (1997), Brain aging: Changes in the nature of information coding by the hippocampus. Journal of Neuroscience, 17, 5155-5166.

Ward, M. T., Stoelzel, C. R., \& Markus, E. J. (1999). Hippocampal memory dysfunction during aging may result from an impaired consolidation process: Aged rats do not show retrograde amnesia of contextual fear conditioning. Manuscript submitted for publication.

\section{NOTES}

1. This, as well as the location of the experimenter, was varied in a pseudo-random manner.

2. This was different from the criteria used for defining an arm choice (i.e., proceeding $24 \mathrm{~cm}$ ) or the equivalent of approximately a full body length down an arm.

3 . On the day of the probe trial, their animals received less food on the maze than they did in the nonprobe sessions (one vs. four pellets), and this may have affected the animals' behavior during the second probe trial.

(Manuscript received January 11, 1999; revision accepted for publication August 10,1999.) 